\title{
Staatsbürgerschaft als Ware - von Goldenen Pässen und der Europäischen Union
}

Julien Berger*

Kammergericht Berlin, Berlin, Deutschland

julien.berger@posteo.de

$\begin{array}{lr}\text { Abstract } & 1033\end{array}$

Keywords $\quad 1034$

I. Einleitung 1034

II. Konzept der Staatsangehörigkeit 1036

1. Bedeutung der Staatsangehörigkeit im nationalen Recht und Völkerrecht 1036

2. Anknüpfungspunkte für die Staatsangehörigkeit 1039

III. Die Staatsbürgerschaft in der EU und die Unionsbürgerschaft 1042

1. Verhältnis zur nationalen Staatsangehörigkeit 1042

2. Inhalt der Unionsbürgerschaft 1044

$\begin{array}{lr}\text { IV. Goldene Pässe } & 1046\end{array}$

1. Überblick 1046

2. Goldene Pässe in der Europäischen Union 1049

V. Vereinbarkeit mit dem Europarecht? 1051

1. Grundsatz der loyalen Zusammenarbeit 1053

2. Grenzen Europäischer Integration 1055

$\begin{array}{ll}\text { VI. Ausblick } & 1057\end{array}$

Summary: Citizenship as a Commodity - of Golden Passports and the European Union 1058

\begin{abstract}
"Goldene Pass“-Programme gewinnen weltweit zunehmend an Popularität. Dieser Trend hat inzwischen auch die Europäische Union erreicht und droht nun in einigen EU-Staaten zu einer teilweisen Kommerzialisierung der Staatsangehörigkeit und damit auch der Unionsbürgerschaft zu führen. Der Beitrag untersucht die Fortentwicklung nationalen Staatsangehörigkeitsrechts durch "Goldene Pässe“ und widmet sich der Frage der Vereinbarkeit entsprechender Programme mit dem Europarecht. Dabei wird deutlich, wie schwer die mitgliedstaatliche Souveränität in Staatsangehörigkeitsfragen mit dem europarechtlichen Grundsatz der loyalen Zusammenarbeit in Einklang zu bringen ist.
\end{abstract}

* Dr., M.Jur. (Oxon), Rechtsreferendar am Kammergericht Berlin. Der Verfasser dankt Prof. Dr. Andreas Zimmermann sowie den anonymen ReviewerInnen des Beitrags herzlich für ihre wertvollen Anmerkungen zum Manuskript. 


\section{Keywords}

Goldene Pässe - Citizenship by Investment - Unionsbürgerschaft - Staatsangehörigkeit - Nottebohm - Micheletti

\section{Einleitung}

Die Staatsangehörigkeit ${ }^{1}$ stellt das formale rechtliche und politische Band zwischen den Bürgern eines Staates und dem staatlich organisierten Gemeinwesen dar. Erst durch diese kann der Einzelne die Rechte und Pflichten, die mit der Zugehörigkeit zu einer politischen Gemeinschaft einhergehen, erwerben und wahrnehmen. Umso beachtlicher sind die jüngeren Entwicklungen der Kommodifizierung der Staatsangehörigkeit, denn eine Vielzahl von Staaten bietet die Möglichkeit, ihre Staatsangehörigkeit im Rahmen sog. „Goldener Pass“-Programme (auch bekannt als „Investor-Citizen-Schemes“ oder „Citizenship-by-Investment“-Programme) käuflich zu erwerben. Lange Zeit waren solche Programme vor allem auf kleine Inselstaaten - vornehmlich in der Karibik - konzentriert, doch die Möglichkeit, hierdurch den Staatshaushalt aufzubessern und Investitionen in die eigene Volkswirtschaft anzuziehen, hat in den letzten Jahren zu ihrer rasanten Verbreitung beigetragen. Dieser Trend hat inzwischen auch die Europäische Union (EU) erreicht und droht in einigen EU-Staaten zu einer teilweisen Kommerzialisierung der Staatsangehörigkeit zu führen. Im Oktober 2020 hat die EU-Kommission nun wegen solcher Programme Vertragsverletzungsverfahren gegen Malta und Zypern eingeleitet. ${ }^{2}$ „Goldene Pass“-Programme sind dabei von sog. „Goldenen Visa“ zu unterscheiden. Letztere ermöglichen gegen bestimmte Geldzah-

1 Dieser Aufsatz unterscheidet nicht zwischen den häufig synonym verwendeten Begriffen der Staatsangehörigkeit und der Staatsbürgerschaft. Im Folgenden wird der neutralere Begriff der Staatsangehörigkeit verwendet. Dem deutschen Grundgesetz ist eine Unterscheidung zwischen einer passiven Staatsangehörigkeit und einer aktiven, personal enger definierten, mit politischen Mitwirkungsrechten einhergehenden Staatsbürgerschaft fremd. Die deutsche Verfassungsordnung kennt allein die "Staatsangehörigkeit", vgl. auch Thomas Giegerich, in: Theodor Maunz/Günter Dürig, Grundgesetz, 92. EL August 2020, München: C. H. Beck 2021, Art. 16 Abs. 1, Rn. 93. Anders hingegen die Weimarer Reichsverfassung von 1919 in ihren Art. 109 und 110. Die aktuelle österreichische Verfassung verwendet in Art. 6 Abs. 1 des Bundes-Verfassungsgesetzes den Begriff der „Staatsbürgerschaft“, wie auch die Verfassung der Deutschen Demokratischen Republik von 1968 in ihrem damaligen Art. 19 Abs. 4. Art. 37 und 38 der aktuellen Bundesverfassung der Schweizerischen Eidgenossenschaft sprechen schließlich von „Schweizerbürgerin“ und „Schweizerbürger“ bzw. „Bürgerrechten“.

2 EU-Kommission Presse-Erklärung vom 20.10.2020, abrufbar unter <https://ec.europa.eu/ commission/presscorner/detail/de/ip_20_1925> (12.10.2021). Siehe auch, Redaktion beck-aktu ell, Zyperns Präsident rechtfertigt Vergabe „Goldener Pässe”, 3.2.2021, becklink 2018769. 
lungen oder Investitionen privilegierte Aufenthaltsrechte in einem bestimmten Land und in vielen EU-Mitgliedstaaten auch Reisefreiheit im gesamten Schengenraum, ${ }^{3}$ ohne jedoch zu einer Verleihung der Staatsangehörigkeit zu führen.

"Goldene Pässe“ werfen eine Vielzahl von Fragen auf, die bis an den Kern des Konzepts der Staatsangehörigkeit heranreichen, denn eine „erkaufte" Staatsangehörigkeit entbindet ihre Erwerber von dem Erfordernis einer gewachsenen Verbindung zum neuen Heimatland. Die finanzielle Investition wird zum Ersatz für die Zugehörigkeit zu einer politischen Gemeinschaft, die Staatsangehörigkeit zur marktfähigen Ware. Die Zuweisung eines monetären Wertes und ihre Handelbarkeit kann dabei aber auch zur „Entwertung" ihres immateriellen Wertes führen, der ihr von der einheimischen Bevölkerung zugeschrieben wird, ähnlich wie das Konzept des Erbadels durch den Verkauf aristokratischer Titel, etwa im Wege der Adoption, bedroht sein könnte. ${ }^{4}$ Während eine solche potenzielle Verwässerung der Staatsangehörigkeit allenfalls und in erster Linie ein "nationales Problem“ darstellt, ist die Situation innerhalb der EU aufgrund der gemeinsamen Unionsbürgerschaft und des gemeinsamen „Raums der Freiheit der Sicherheit und des Rechts ohne Binnengrenzen, in dem [...] der freie Personenverkehr gewährleistet ist" ${ }^{\text {"5 }}$ eine andere. Der vorliegende Aufsatz widmet sich daher der Frage "Goldener Pässe“ im Kontext der Europäischen Union.

Nach einer kurzen Einführung in das Konzept der Staatsangehörigkeit und ihrer klassischen Anknüpfungspunkte im nationalen und internationalen Recht (II.) sowie der besonderen Situation innerhalb der Europäischen Union mit ihrer Unionsbürgerschaft (III.), beleuchtet der Aufsatz beispielhaft das Konzept der „Goldenen Pässe“ (IV.) und befasst sich sodann mit der Frage ihrer Vereinbarkeit mit dem Europarecht (V.).

3 Entsprechende Visa bietet die große Mehrzahl an EU-Mitgliedstaaten an, vgl. Bericht der EU-Kommission: Staatsbürgerschaftsregelungen und Aufenthaltsregelungen für Investoren in der EU, 23.1.2019, COM(2019) 12 final, $9 \mathrm{ff}$.

4 Vgl. auch Kai A. Konrad/Ray Rees, Passports for Sale: The Political Economy of Conflict and Cooperation in a Meta-Club, European Journal of Political Economy 62 (2020), 2.

5 Art. 3 Abs. 2 EUV. 


\section{Konzept der Staatsangehörigkeit}

\section{Bedeutung der Staatsangehörigkeit im nationalen Recht und Völkerrecht}

Nach der klassischen Definition Georg Jellineks ist der Staat „die mit ursprünglicher Herrschermacht ausgerüstete Verbandseinheit sesshafter Menschen". 6 Anders formuliert, besteht ein Staat aus den drei Elementen Staatsgebiet, Staatsvolk und Staatsgewalt. Das Staatsvolk ist dabei aus völkerrechtlicher Sicht keine ethnische Größe, sondern ist definiert durch das formale Band der Staatsangehörigkeit, also der wechselseitigen Rechts- und Pflichtenbeziehung zwischen einem Staat und seinen Bürgern. ${ }^{7}$ Mit der Staatsangehörigkeit bestimmt der Staat also sein Volk. Die Staatsangehörigkeit stellt dabei eine Form „legalisierter Diskriminierung“8 dar, denn mit Blick auf die Gewährung von Rechten und staatlichen Leistungen differenzieren Staaten in ihren Rechtsordnungen kategorisch auf Grundlage des als Staatsangehörigkeit definierten Zugehörigkeitskriteriums. ${ }^{9}$ Ihren Ursprung hat diese Differenzierung darin, dass die Staatsangehörigkeit als konstitutives Element des politischen Gemeinwesens, also letztlich der Staatlichkeit angesehen wird. Für den Internationalen Gerichtshof (IGH) ist die Staatsangehörigkeit daher rechtlicher Ausdruck der Tatsache, dass diejenigen, denen sie entweder unmittelbar durch Gesetz oder durch hoheitlichen Akt verliehen worden ist, enger mit der Bevölkerung dieses Staates als mit der eines jeden anderen Staates verbunden sind. ${ }^{10}$ Ähnlich definiert sie der Europäische Gerichtshof (EuGH), für den die Staatsangehörigkeit als Band zwischen dem Staat und seinen Staatsbürgern Ausdruck der besonderen Verbundenheit und gegenseitigen Loyalität ist. ${ }^{11}$

6 Georg Jellinek, Das Recht des modernen Staates, 1. Band - Allgemeine Staatslehre, 2. Aufl., Berlin: O. Häring Verlag 1905, 193.

7 Vgl. Andreas von Arnauld, Völkerrecht, 3. Aufl., Heidelberg: C. F. Müller 2016, Rn. 82.

8 Andreas Wimmer, Ethnic Boundary Making: Institutions, Power, Networks, New York: Oxford University Press 2013, 74.

9 Vgl. Maarten Vink, Comparing Citizenship Regimes, in: Ayelet Shachar/Rainer Bauböck/ Irene Bloemraad/Maarten Vink, The Oxford Handbook of Citizenship, Oxford: Oxford University Press 2017, 221-245 (221 f.).

10 IGH, Nottebohm Case (Liechtenstein v. Guatemala), Second Phase, Urteil v. 6.4.1955, ICJ Reports 1955, 4, (23).

11 EuGH, Urteil v. 2.3.2010, Rs. C-135/08, ECLI:EU:C:2010:104, Rn. 51 - Rottmann; EuGH, Urteil v. 12.3.2019, Rs. C-221/17, ECLI:EU:C:2019:189, Rn. 33 - Tjebbes. 
Während in der globalisierten Welt die staatliche Souveränität in vielen Bereichen eher an Bedeutung verliert, ${ }^{12}$ bleibt das Staatsangehörigkeitsrecht damit gewissermaßen ureigenster Ausdruck staatlicher Souveränität bzw. „die letzte Bastion in der Zitadelle der Souveränität“. ${ }^{13}$ In den Worten des IGH:

„Es ist Sache [...] jedes souveränen Staates, die Regeln für den Erwerb seiner Staatsangehörigkeit durch seine eigene Gesetzgebung zu bestimmen und diese Staatsangehörigkeit durch Einbürgerung zu verleihen." ${ }^{14}$

Dieses Verständnis spiegelt sich auch in Art. 1 des Haager Übereinkommen über einzelne Fragen beim Konflikt von Staatsangehörigkeitsgesetzen ${ }^{15}$ aus dem Jahre 1930 wider:

„It is for each State to determine under its own law who are its nationals. This law shall be recognised by other States in so far as it is consistent with international conventions, international custom, and the principles of law generally recognised with regard to nationality."

sowie im fast wortgleichen - vom Europarat geschlossenen - Art. 3 des Europäischen Übereinkommens über die Staatsangehörigkeit ${ }^{16}$ von 1997:

(1) Jeder Staat bestimmt nach seinem eigenen Recht, wer seine Staatsangehörigen sind.

(2) Dieses Recht ist von den anderen Staaten anzuerkennen, soweit es mit anwendbaren internationalen Übereinkommen, dem Völkergewohnheitsrecht und den mit Bezug auf die Staatsangehörigkeit allgemein anerkannten Rechtsgrundsätzen in Einklang steht.

In seiner Entscheidung Qatar v. United Arab Emirates hat der IGH mit Blick auf das Internationale Übereinkommen zur Beseitigung jeder Form von Rassendiskriminierung (ICERD) ${ }^{17}$ jüngst die Unterscheidung zwischen Staatsangehörigkeit (nationality) und nationaler Herkunft (national origin) hervorgehoben. Während aus Sicht des Gerichtshofs erstere ein veränderbares rechtliches Attribut darstellt, das im Ermessen des Staates liegt, be-

12 Vgl. m.w. N. Heike Krieger/Georg Nolte, The International Rule of Law - Rise or Decline? Points of Departure, KFG Working Paper Series, Nr. 1, Berlin Potsdam Research Group “The International Rule of Law - Rise or Decline?”, Berlin, Oktober 2016.

13 Peter J. Spiro, A New International Law of Citizenship, AJIL 105 (2011), 694-746 (746).

14 IGH, Nottebohm (Fn. 10), 20. [Übersetzung vom Autor].

15 Convention on Certain Questions relating to the Conflict of Nationality Laws v. 12.4. 1930, League of Nations, Treaty Series, Bd. 179, 89.

16 European Convention on Nationality v. 6.11.1997, ETS Nr. 166.

17 International Convention on the Elimination of All Forms of Racial Discrimination v. 21.12.1965, UNTS, 660, 195. 
schreibt letztere die unveränderliche Bindung einer Person an eine nationale Gruppe bei der Geburt. ${ }^{18}$ Die Staatsangehörigkeit wird daher von der nationalen Herkunft nicht mitumfasst. ${ }^{19}$

Die Staatsangehörigkeit begründet Rechte und Pflichten innerhalb eines Staates, wie etwa das aktive und passive Wahlrecht in Demokratien, ${ }^{20}$ den Zugang zu öffentlichen Ämtern ${ }^{21}$ oder auch die Pflicht zum Militärdienst ${ }^{22}$. Sie ist der Anknüpfungspunkt für konsularische Betreuung und diplomatischen Schutz. ${ }^{23}$ Die staatliche Unterstützung durch Sozialsysteme, aber auch die Inanspruchnahme bestimmter Grundrechte, ${ }^{24}$ knüpfen häufig an die Staatsangehörigkeit an. Ausländer unterfallen hingegen in der Regel einem nationalen ausländerrechtlichen oder völkerrechtlichen Sonderregime. Mit hinreichendem Grund können sie aus einem Staat ausgewiesen werden und ihr Heimatstaat ist grundsätzlich verpflichtet, sie aufzunehmen. ${ }^{25}$ Schließlich knüpft die Ausübung staatlicher Gerichtsbarkeit etwa im Strafrecht häufig auch an die Staatsangehörigkeit an, selbst in Bezug auf im Ausland begangene strafbare Handlungen. ${ }^{26}$

18 IGH, Application of the International Convention on the Elimination of all Forms of Racial Discrimination (Qatar v. United Arab Emirates), Preliminary Objections, Urteil v. 4.2. 2021, Rn. 81.

19 IGH, Qatar v. United Arab Emirates (Fn. 18), Rn. 88, 97, 105. Anderer Ansicht Judge Robinson, für den sich die nationale Herkunft nicht nur auf den Ort bezieht, aus dem die Vorfahren einer Person stammen, sondern auch auf den Ort, an dem diese selbst geboren wurde. Daher könne die nationale Herkunft auch die Staatsangehörigkeit umfassen. Denn der Ort der Geburt könne sowohl die Staatsangehörigkeit als auch die nationale Herkunft begründen. IGH, Qatar v. United Arab Emirates (Fn. 18), Dissenting Opinion of Judge Robinson, Rn. 7 f. Ähnlicher Ansicht Judge Bhandari, für den sich die "nationale Herkunft“ auf die Zugehörigkeit einer Person zu einem Land oder einer Nation bezieht. Diese Zugehörigkeit könne historisch oder durch Abstammung begründet sein, oder durch den rechtlichen Status der Staatsangehörigkeit oder der nationalen Zugehörigkeit bestätigt werden. Daher sei die Staatsangehörigkeit einer Person in jedem Fall von dem weiter gefassten Begriff „nationale Herkunft" umfasst, insbesondere in Staaten, die ihre Staatsangehörigkeit an die Abstammung von anderen Staatsangehörigen (ius sanguini) knüpfen. IGH, Qatar v. United Arab Emirates (Fn. 18), Dissenting Opinion of Judge Bhandari, Rn. 10, 11, 20.

20 Vgl. in Deutschland, Art. 38 Abs. 1 S. 1 GG und Art. 28 Abs. 1 S. 2 GG.

21 Vgl. in Deutschland, Art. 30 GG.

22 Vgl. in Deutschland, Art. 12 a Abs. 1 GG.

23 Christian Hillgruber, in: Volker Epping/Christian Hillgruber (Hrsg.), BeckOK Grundgesetz, 45. Edition, Stand: 15.11.2020, München: C. H. Beck 2021, Art. 166, Rn. 2.

24 In Deutschland wird etwa zwischen „Jedermannrechten“ und „Deutschenrechten“ unterschieden, vgl. Art. 8, 9, 12, 33 und 38 GG.

25 Vgl. James Crawford, Brownlie's Principle of Public International Law, 9. Aufl., Oxford: Oxford University Press 2019, $495 \mathrm{f}$.

26 Siehe beispielsweise $\mathbb{} 7 \mathrm{StGB}$, wonach das deutsche Strafrecht u. a. auch für Taten gilt, die im Ausland gegen einen Deutschen oder von einem Deutschen begangen worden sind. Siehe auch, Hillgruber (Fn. 23), Rn. 2. 
Aufgrund der immensen Bedeutung der Staatsangehörigkeit im Rechtsverkehr, beinhaltet die Allgemeine Erklärung der Menschenrechte ${ }^{27}$ aus dem Jahre 1948 in ihrem Art. 15 einerseits das Recht auf eine Staatsangehörigkeit, andererseits das Verbot des willkürlichen Entzugs der Staatsangehörigkeit und der Versagung des Wechsels der Staatsangehörigkeit. Das Recht auf die Staatsangehörigkeit findet sich auch in völkerrechtlichen Verträgen, etwa in Art. 5 lit. c) iii) ICERD oder Art. 7 Abs. 1 der Kinderrechtskonvention der Vereinten Nationen. ${ }^{28}$ Es dient in erster Linie dem Schutz vor Staatenlosigkeit. Das Recht auf den Wechsel der Staatsangehörigkeit, wie es sich aus Art. 15 Abs. 2 Fall 2 der Allgemeinen Erklärung der Menschenrechte ergibt, verbietet es hingegen einem früheren Staat sich willkürlich zu weigern, eine Person aus ihrer (bestehenden) Staatsangehörigkeit zu entlassen. ${ }^{29}$ Hieraus ergibt sich aber gerade kein Anspruch auf den Erwerb einer neuen Staatsangehörigkeit. ${ }^{30}$

\section{Anknüpfungspunkte für die Staatsangehörigkeit}

Die Staatsangehörigkeitskonzepte der verschiedenen Staaten ähneln sich in bemerkenswerter Weise. Der Erwerb der Staatsbürgerschaft erfolgt in der Regel durch Geburt oder Einbürgerung, ${ }^{31}$ wobei erstere klassischerweise an den Ort der Geburt (ius soli) oder die Abstammung von anderen Staatsangehörigen (ius sanguinis) anknüpft. ${ }^{32}$ Die Einbürgerung hingegen begründet die Staatsangehörigkeit zu einem späteren Zeitpunkt durch einen freiwilligen hoheitlichen Akt auf Grundlage nationaler Einbürgerungsregeln. Diese spiegeln meist das gesellschaftliche Verständnis des Staats-

27 Universal Declaration of Human Rights, UNGA Resolution 217 A (III) v. 10.12.1948.

28 Convention on the Rights of the Child v. 20.11.1989, UNTS 1577, 3.

29 Anne Peters, Passportisation: Risks for International Law and Stability - Part I, EJIL: Talk!, 9.5.2019.

30 So auch Mónika Ganczer, The Right to a Nationality as a Human Right?, Hungarian Yearbook of International Law and European Law 15 (2014), 15-34 (18).

31 Eine Übersicht der nationalen Voraussetzungen des Staatsangehörigkeitserwerbs findet sich in der Datenbank "Global Database on Modes of Acquisition of Citizenship“, abrufbar unter <http://globalcit.eu/acquisition-citizenship/> (12.10.2021).

32 In der Bundesrepublik ist - als Besonderheit des deutschen Verfassungsrechts neben den deutschen Staatsangehörigen noch der Begriff des „Statusdeutschen“ eingeführt. Gem. Art. 116 I GG werden bestimmte, von Verfolgungs- und Vertreibungsmaßnahmen betroffene und ins alte Reichsgebiet geflüchtete deutsche Volkszugehörige und deren Familien als sog. Statusdeutsche den deutschen Staatsangehörigen gleichgestellt. Vgl. Hillgruber (Fn. 23), Rn. 5 ff.; Winfried Kluth, in: Winfried Kluth/Andreas Heusch (Hrsg.), BeckOK Ausländerrecht, 28. Edition, Stand: 1.1.2021, München: C. H. Beck 2021, Art. 116 GG, Rn. 6. 
volks wieder und beantworten somit die Frage nach einem gesellschaftlichen „Wir“ und nach den erwünschten Qualitäten möglicher zukünftiger Mitglieder der Gemeinschaft. ${ }^{33}$ Daraus zieht der IGH den treffenden Schluss:

„Die Einbürgerung ist keine Angelegenheit, die man auf die leichte Schulter nehmen sollte. Sie anzustreben und zu erreichen ist nicht etwas, das im Leben eines Menschen häufig vorkommt. Es bedeutet, ein Band der Zugehörigkeit zu brechen und ein neues Band der Zugehörigkeit aufzubauen." ${ }^{34}$

Für eine Einbürgerung wird meist auf eine nachträglich erwachsene Verbundenheit zu einem Staat bzw. einer Gesellschaft abgestellt, die in der Regel einen Wohnsitz von gewisser Dauer im Einbürgerungsstaat voraussetzt oder an die Ehe mit einem Staatsangehörigen anknüpft. ${ }^{35}$ So sieht etwa $\$ 10$ Abs. 1 des deutschen Staatsangehörigkeitsgesetz eine Einbürgerung auf Antrag vor, wenn ein Ausländer seit acht Jahren rechtmäßig seinen gewöhnlichen Aufenthalt in der Bundesrepublik hat und sich u.a. zur freiheitlichdemokratischen Grundordnung bekennt, ein unbefristetes Aufenthaltsrecht besitzt, den Lebensunterhalt für sich und seine Familienangehörigen bestreiten kann, nicht vorbestraft ist und über ausreichende Kenntnisse sowohl der deutschen Sprache als auch über die Rechts- und Gesellschaftsordnung sowie die Lebensverhältnisse in Deutschland verfügt. Art. 116 Abs. 2 S. 1 Grundgesetz (GG) sieht darüber hinaus vor, dass frühere deutsche Staatsangehörige, denen zwischen dem 30. Januar 1933 und dem 8. Mai 1945 die Staatsangehörigkeit aus politischen, rassistischen oder religiösen Gründen entzogen worden ist, und ihre Abkömmlinge auf Antrag wieder einzubürgern sind. ${ }^{36}$

Die Verbundenheit kann aber auch an andere Kriterien anknüpfen. In Österreich kann etwa eine Einbürgerung auch ohne Wohnsitz oder längere Verweildauer im Land gem. $\mathbb{1} 10$ Abs. 6 des österreichischen Staatsbürgerschaftsgesetzes erfolgen, wenn „die Verleihung der Staatsbürgerschaft wegen der vom Fremden bereits erbrachten und von ihm noch zu erwartenden außerordentlichen Leistungen im besonderen Interesse der Republik liegt" - etwa in den Bereichen Kultur, Wissenschaft oder Sport. Eine solch außerordentliche Leistung kultureller Natur wurde beispielsweise im Fall der Einbürgerung der Opernsängerin Anna Netrebko angenommen. ${ }^{37}$ In

33 Vgl. auch Liav Orgad, Naturalization, in: Shachar/Bauböck/Bloemraad/Vink (Fn. 9), 337-357 (337f.).

34 IGH, Nottebohm (Fn. 10), 24. [Übersetzung vom Autor].

35 Siehe m. w. N. Crawford (Fn. 25), 497 f.; siehe auch, EU-Kommission (Fn. 3), 6.

36 Ausführlich hierzu, Kluth (Fn. 32), Rn. $9 \mathrm{ff}$.

37 Peter Hilpold, Die verkaufte Unionsbürgerschaft, NJW 67 (2014), 1071-1074 (1072). 
Israel ermöglicht das „Rückkehrgesetz“ vom 5. Juli $1950^{38}$ jeder jüdischen Person nach der „Alija“, also der Rückkehr bzw. der Auswanderung nach Israel, die israelische Staatsbürgerschaft anzunehmen. ${ }^{39}$ Ein anderes Beispiel ist schließlich der - zugegebenermaßen auch in anderen Bereichen untypische - Vatikanstaat, der für die Staatsangehörigkeit allein an die Ausübung einer kirchlichen Funktion für die Dauer des Wohnsitzes in der Vatikanstadt anknüpft. ${ }^{40}$

Die Vergabe der Staatsangehörigkeit ist also eine unilaterale staatliche Entscheidung. Andere Staaten sind jedoch nicht uneingeschränkt zur Anerkennung einer fremden Staatsangehörigkeit verpflichtet, wie der IGH in seiner berühmten Nottebohm-Entscheidung mit Blick auf diplomatischen Schutz aufzeigte. ${ }^{41}$ Darin unterscheidet der Gerichtshof die innerstaatlichen und völkerrechtlichen Wirkungen der Staatsangehörigkeit. ${ }^{42}$ Entscheidend für die Anerkennung eines auf der Staatsangehörigkeit beruhenden diplomatischen Schutzes ist danach, eine ecbte tiefgehende Verbindung („genuine link“) zu dem die Staatsangehörigkeit vergebenden Staat. Denn durch die Ausübung eines solchen Schutzrechtes begibt sich der Staat auf die Ebene des Völkerrechts, welches letztlich bestimmt, ob ein Staat dazu befugt ist, dieses Recht auszuüben. ${ }^{43}$ Fehlt eine solche Verbindung müssen andere Staaten diese Staatsangehörigkeit im Rahmen des gegen sie geltend gemachten diplomatischen Schutzes nicht anerkennen. ${ }^{44}$

38 Law of Return, 5.7.1950, abrufbar unter <https://mfa.gov.il/> (12.10.2021).

39 Erst 1970 wurde dieses Kriterium dann näher definiert: „Für die Zwecke dieses Gesetzes bedeutet Jude eine Person, die von einer jüdischen Mutter geboren wurde oder zum Judentum übergetreten ist und die keiner anderen Religionsgemeinschaft angehört." Siehe ausführlich hierzu, Michael Brenner, Israel - Traum und Wirklichkeit des jüdischen Staates, 2. Aufl., München: C. H.Beck 2017, $144 \mathrm{ff}$.

40 Art. 1 Legge N. CVVI sulla cittadinanza, la residenza e l'acesso v. 22.2.2011; vgl. auch Kay Hailbronner, in: Kay Hailbronner/Hans-Georg Maaßen/Jan Hecker/Marcel Kau (Hrsg.), Staatsangehörigkeitsrecht, 6. Aufl., München: C. H. Beck 2017, \25 StAG, Rn. 17.

41 Siehe hierzu unter IV. 1.

42 Alexander N. Makarov, Das Urteil des Internationalen Gerichtshofs im Fall Nottebohm, ZaöRV 16 (1955), 407-426 (411, 413); José-Miguel Bello y Villarino, If Mr Nottebohm Had a Golden Passport, Cambridge International Law Journal 9 (2020), 76-95 (84). Ähnlich auch, Iran-US-Claims Tribunal, Iran v. United States, Urteil v. 6.4.1984, Case A/18-FT, Rn. 37.

43 Erwin H. Loewenfeld, Der Fall Nottebohm, AVR 5 (1956), 387-410 (390).

44 IGH, Nottebohm (Fn. 10), 26. 


\section{Die Staatsbürgerschaft in der EU und die Unionsbürgerschaft}

\section{Verhältnis zur nationalen Staatsangehörigkeit}

Die Unionsbürgerschaft begründet ein Staatsbürgerschaftskonzept jenseits des Nationalstaates. ${ }^{45}$ Für den EuGH ist sie dazu bestimmt, „der grundlegende Status der Angehörigen der Mitgliedstaaten zu sein, " ${ }^{46}$ der es denjenigen unter ihnen, die sich in der gleichen Situation befinden, erlaubt, unabhängig von ihrer Staatsangehörigkeit die gleiche rechtliche Behandlung zu genießen. ${ }^{47}$ Insoweit handelt es sich um eine „staatsübergreifende Bürgerschaft, die den Angehörigen eines Mitgliedstaats verschiedene Rechte in den übrigen Mitgliedstaaten verleiht, darunter insbesondere das Recht, sich im Hoheitsgebiet der Mitgliedstaaten frei zu bewegen und aufzuhalten, sowie das Recht auf Gleichbehandlung, auch im Verhältnis zur Union selbst““.48

Die Unionsbürgerschaft wurde 1992 in Art. 8 Abs. 1 des Maastricht Vertrags über die Europäische Union (EUV) eingeführt. Gemäß der gleichlautenden Art. 9 S. 3 EUV und Art. 20 Abs. 1 S. 3 Vertrag über die Arbeitsweise der Europäischen Union (AEUV) tritt sie zur nationalen Staatsbürgerschaft hinzu, ersetzt diese aber nicht. Die Unionsbürgerschaft kann daher auch nicht isoliert aufgegeben und von Staatenlosen oder Drittstaatsangehörigen auch nicht selbständig - ohne eine mitgliedstaatliche Staatsangehörigkeit erworben werden. ${ }^{49}$ Sie ist allein von dem Willen der Mitgliedstaaten abge-

45 Es geht dabei deutlich weiter als das "Citizenship" des "Commonwealth of Nations“, vgl. Robert R. Wilson/Robert E. Clute, Commonwealth Citizenship and Common Status, AJIL 57 (1963), 566-587; Kay Hailbronner in: Kay Hailbronner/Hans-Georg Maaßen/Jan Hecker/ Marcel Kau (Hrsg.), Staatsangehörigkeitsrecht, 6. Aufl., München: C. H. Beck 2017, H. Deutsche Staatsangehörigkeit und Unionsbürgerschaft, Rn. 15. Ähnlichkeiten bestehen hingegen mit dem in Art. 3 der Verfassung des Norddeutschen Bundes vom 16.4.1867 garantierten „gemeinsamen Indigenat“. Der Norddeutsche Bund konnte noch nicht als Nationalstaat verstanden werden, hatte jedoch eine bundeseinheitliche Staatsangehörigkeit, deren Erwerb und Verlust ausschließlich durch das Recht der Teilstaaten vermittelt wurde. Das Indigenat schuf aber keine eigenen bundeseinheitlichen Rechte und Pflichten, sondern garantierte eine Inländergleichbehandlung der verschiedenen Staatsbürger der Teilstaaten und deren Freizügigkeit im gesamten Bundesgebiet des Norddeutschen Bundes. Vgl. Hailbronner (Fn. 45), Rn. 15. Vgl. auch die von den sowjetischen Teilrepubliken abgeleitete Staatsbürgerschaft der Sowjetunion, Art. 21 der Verfassung der Union der Sozialistischen Sowjetrepubliken vom 5.12.1936.

46 M. w. N. EuGH, Rottmann (Fn. 11), Rn. 43.

47 EuGH, Urteil v. 20.9.2001, Rs. C-184/99, ECLI:EU:C:2001:458, Rn. 31 - Grzelczyk.

$48 \mathrm{EuGH}$, Schlussanträge des Generalanwalts Maduro v. 30.9.2009, Rs. C-135/08, ECLI: EU:C:2009:588, Rn. 16 - Rottmann.

49 Thomas Giegerich, in: Reiner Schulze/André Janssen/Stefan Kadelbach (Hrsg.), Europarecht, 4. Aufl., Baden-Baden: Nomos 2020, $\$ 9$ Unionsbürgerschaft, Rn. 23. 
leitet und konstituiert folglich (noch) kein Unionsvolk, das als sich selbst verfassendes Rechtssubjekt zur eigenen Selbstbestimmung berufen wäre. ${ }^{50}$ Die Abhängigkeit vom nationalen Status zeigt sich auch in der Tatsache, dass alle Briten in Folge des Brexits ihre Unionsbürgerschaft verloren haben. Aus der Unionsbürgerschaft könnte also erst dann eine europäische Staatsangehörigkeit werden, wenn der Staatenverbund der EU in einen Bundesstaat umgewandelt würde. ${ }^{51}$

Die mitgliedstaatliche Staatsangehörigkeit wiederum richtet sich nach dem nationalen Recht jedes einzelnen Mitgliedstaates. ${ }^{52}$ Bereits im Jahr 1990 hatte der EuGH jedoch der Ausübung dieses Rechts einen - nicht näher konkretisierten - europarechtlichen Rahmen zugewiesen und in Micheletti festgestellt:

„Die Festlegung der Voraussetzungen für den Erwerb und den Verlust der Staatsangehörigkeit unterliegt nach dem internationalen Recht der Zuständigkeit der einzelnen Mitgliedstaaten; von dieser Zuständigkeit ist unter Beachtung des Gemeinschaftsrechts Gebrauch zu machen." ${ }^{53}$

Diese Einschränkung „unter Beachtung des Gemeinschaftsrechts“ schien den europäischen Institutionen eine Rolle mit Blick auf die Staatsangehörigkeit der Mitgliedstaaten zu eröffnen. Daher schlug das Europäische Parlament 1991 in seiner „Entschließung zur Unionsbürgerschaft“ vor, in den Vertrag über die Europäische Union u. a. aufzunehmen, dass diese „gewisse einheitliche Bedingungen für den Erwerb oder den Verlust der Staatsbürgerschaft der Mitgliedstaaten“ festlegen könne. ${ }^{54}$ Dieser Vorschlag wurde jedoch nicht umgesetzt.

$50 \mathrm{Vgl}$. BVerfGE 123, 267 (Rn. 346) - Lissabon.

51 Vgl. Hailbronner (Fn. 45), Rn. 11.

52 EuGH, Rottmann (Fn. 11), Rn. 39, 41. Siehe auch die „Erklärung zur Staatsangehörigkeit eines Mitgliedstaats“ (Erklärung Nr. 2) zum Vertrag von Maastricht v. 7.2.1992, wonach „die Frage, welchem Mitgliedstaat eine Person angehört, allein durch Bezug auf das innerstaatliche Recht des betreffenden Mitgliedstaates geregelt wird“. Siehe hierzu auch Andreas Zimmermann, Europäisches Gemeinschaftsrecht und Staatsangehörigkeitsrecht der Mitgliedstaaten unter besonderer Berücksichtigung der Probleme mehrfacher Staatsangehörigkeit, EuR 31 (1995), 54-70 (55 f.).

53 EuGH, Urteil v. 7.7.1992, Rs. C-369/90, ECLI:EU:C:1992:295, Rn. $10-$ Micheletti. Siehe zur Micheletti-Entscheidung auch Christoph Schönberger, in: Eberhard Grabitz/Meinhard Hilf/Martin Nettesheim (Hrsg.), Das Recht der Europäischen Union, 71. EL August 2020, München: C. H. Beck 2020, Art. 20 AEUV, Rn. 40.

54 Europäisches Parlament, Entschließung zur Unionsbürgerschaft, 16.12.1991, Amtsblatt der Europäischen Union Nr. C 326/205, A3-300/91. Vgl. auch Willem Maas, European Governance of Citizenship and Nationality, Journal of Contemporary European Research 12 (2016), 533-551 (535). 
Die Festlegung der Voraussetzungen für Erwerb und Verlust der Staatsangehörigkeit verbleibt also weiterhin bei den Mitgliedstaaten, die davon jedoch - nach inzwischen ständiger Rechtsprechung des EuGH - unter Beachtung des Unionsrechts Gebrauch zu machen haben. ${ }^{55}$ Das bedeutet,

„dass im Fall von Unionsbürgern die Ausübung dieser Zuständigkeit, soweit sie die von der Rechtsordnung der Union verliehenen und geschützten Rechte berührt [...] der gerichtlichen Kontrolle im Hinblick auf das Unionsrecht unterliegt. " ${ }^{6}$

Denn aufgrund der Verknüpfung von Unionsbürgerschaft und Staatsangehörigkeit führt eine mitgliedstaatliche Entscheidung über Verleihung und Entziehung der Staatsangehörigkeit auch stets zu einer entsprechenden Entscheidung bezüglich der Unionsbürgerschaft, wenn nicht ausnahmsweise eine doppelte Staatsbürgerschaft ${ }^{57}$ innerhalb der Union besteht..$^{58}$

Die Grenzen sind jedoch noch nicht abschließend durch den EuGH bestimmt worden. Einerseits wäre das Unionsrecht zumindest berührt, wenn einem bisherigen Unionsbürger ohne triftigen Grund - etwa der Erschleichung der Staatsangehörigkeit durch Täuschung - durch eine Ausbürgerung der Genuss der Grundfreiheiten entzogen würde. ${ }^{59}$ Andererseits bleibt die Frage unionsrechtlicher Grenzen bei der Einbürgerung bislang offen. ${ }^{60}$ So ist eine Einbürgerung, etwa zur Integration im Inland lebender Drittstaatsangehöriger, unionsrechtlich unproblematisch. ${ }^{61}$ Inwieweit eine Einbürgerung ohne jegliche Verbundenheit zu einem Mitgliedstaat, etwa aus rein ökonomischen Motiven, europarechtlich zu beanstanden wäre, ist bislang hingegen noch ungeklärt. ${ }^{62}$

\section{Inhalt der Unionsbürgerschaft}

Während die Unionsbürgerschaft zunächst als rein symbolisches Konzept aufgefasst wurde, ${ }^{63}$ das den durch die Grundfreiheiten bereits garan-

55 M. w. N. EuGH, Micheletti (Fn. 53), Rn. 10; EuGH, Rottmann (Fn. 11), Rn. 39, 41; EuGH, Tjebbes (Fn. 11), Rn. $41 \mathrm{ff}$.

56 EuGH, Rottmann (Fn. 11), Rn. 48.

$57 \mathrm{Vgl}$. Art. $14 \mathrm{ff}$. Europäisches Übereinkommen über die Staatsangehörigkeit; siehe auch Oliver Dörr, Nationality, in: Rüdiger Wolfrum (Hrsg.), MPEPIL, Online Edition, Oxford: Oxford University Press 2019, Rn. $21 \mathrm{ff}$.

58 Schönberger (Fn. 53), Rn. 44.

59 EuGH, Rottmann (Fn. 11), Rn. $54 \mathrm{ff}$.

60 Gute Übersicht zu den verschiedenen Positionen in der Literatur bei Schönberger (Fn. 53), Rn. $42 \mathrm{ff}$.

61 Ulrich Forsthof, in: Grabitz/Hilf/Nettesheim (Fn. 53), Art. 45 AEUV, Rn. 19.

62 Siehe hierzu unter V.

63 Für eine gute Übersicht, siehe etwa Kristīne Krūma, EU Citizenship, Nationality and Migrant Status - An Ongoing Challenge, 1. Aufl., Leiden: Brill 2014, 120 f. 
tierten Rechtszustand für Angehörige der EU-Mitgliedstaaten nur erneut bestärkte, entwickelte der EuGH die Unionsbürgerschaft im Laufe der Jahre zu einem eigenständigen Element der Unionsrechtsordnung. Inzwischen wird sie gar als „integrationspolitische Kronjuwelen“64 der EU bezeichnet, die jedem EU-Bürger erlauben, in der ganzen Union von sich sagen zu können: „civis europaeus sum“. 65 Art. 20 Abs. 2 AEUV zählt Rechte der Unionsbürger auf, die in den Art. 21-24 AEUV weiter konkretisiert werden. Darunter das Recht sich gem. Art. 21 AEUV im Hoheitsgebiet der Mitgliedstaaten frei zu bewegen und aufzuhalten, auch ohne einer wirtschaftlichen Betätigung nachzugehen, die sonst zur Eröffnung des Anwendungsbereichs der Grundfreiheiten erforderlich ist. ${ }^{66}$ Unionsbürger besitzen in dem Mitgliedstaat, in dem sie ihren Wohnsitz haben, gem. Art. 22 AEUV das aktive und passive Wahlrecht bei Kommunalwahlen und den Wahlen zum Europäischen Parlament. In Drittstaaten, in denen ihr Heimatstaat nicht vertreten ist, haben sie gem. Art. 23 AEUV Recht auf diplomatischen und konsularischen Schutz durch Behörden eines jeden anderen Mitgliedstaats, unter denselben Bedingungen wie dessen Staatsangehörige. ${ }^{67}$ Schließlich haben sie gem. Art. 24 AEUV ein Petitionsrecht an das Europäische Parlament. Neben diesen kodifizierten Rechten hat der EuGH in seiner Rechtsprechung den Status der Unionsbürgerschaft immer weiter gefasst ${ }^{68}$ und beispielsweise zur Gleichbehandlung bei Sozialleistungen, ${ }^{69}$ zur Korrektur nationalen Namensrechts ${ }^{70}$ und zur Ausweitung von Aufenthaltsrechten Drittstaatsangehöriger ${ }^{71}$ auch bei rein innerstaatlichen Sachverhalten angewendet.

64 Hilpold (Fn. 37), 1073.

65 EuGH, Schlussanträge des Generalanwalts Jacobs v. 9.12.1992, Rs. C-168/91, ECLI:EU: C:1992:504, Rn. 46 - Konstantinidis.

66 Vgl. Martin Nettesheim, in: Grabitz/Hilf/Nettesheim (Fn. 53), Art. 21 AEUV, Rn. 19.

67 Zur Reichweite des sachlichen Schutzbereichs, insbesondere der umstrittenen Frage, inwieweit der Aufnahmestaat neben dem konsularischen auch diplomatischen Schutz durch einen anderen EU-Mitgliedstaat anerkennen muss, siehe ausführlich Armin Hatje, in: Jürgen Schwarze/Ulrich Becker/Armin Hatje/Johann Schoo, EU-Kommentar, 4. Aufl., Baden-Baden: Nomos 2019, Art. 23 AEUV, Rn. $8 \mathrm{ff}$.

68 Gute Übersicht bei Kay Hailbronner/Daniel Thym, Ruiz Zambrano - Die Entdeckung des Kernbereichs der Unionsbürgerschaft, NJW 64 (2011), 2008-2014.

69 EuGH, Grzelczyk (Fn. 47).

70 EuGH, Urteil v. 2.10.2003, Rs. C-148/02, ECLI:EU:C:2003:539 - Garcia Avello.

71 EuGH, Urteil v. 19.10.2004, Rs. C-200/02, ECLI:EU:C:2004:639 - Zhu und Chen; EuGH, Urteil v. 25.7.2008, Rs. C-127/08, ECLI:EU:C:2008:449 - Metock; EuGH, Urteil v. 8.3. 2011, Rs. C-34/09, ECLI:EU:C:2011:124 - Ruiz Zambrano. 


\section{Goldene Pässe}

\section{1. Überblick}

Wie bereits erwähnt, erfolgt die Einbürgerung in der Regel aufgrund einer besonderen Verbundenheit zu einem Staat. Fehlt diese, ist eine Einbürgerung jedoch nicht zwingend ausgeschlossen, wie „Goldene Pass“-Programme auf der ganzen Welt zeigen; vielmehr erlauben immer mehr Staaten, die fehlende Verbundenheit zu ihrem staatlichen Gemeinwesen durch finanzielle Zuwendungen (in Form von Investitionen und/oder Direktzahlungen) zu ersetzen. ${ }^{72}$ Diese Programme begründen somit ein ius argenti, das neben die bisherigen Anknüpfungspunkte des ius soli und ius sanguinis bzw. das Kriterium der engen Verbundenheit tritt. ${ }^{73}$ Die Nachhaltigkeit des Verkaufs von Staatsbürgerschaften erscheint jedoch fraglich. Liegt die reine persönliche Interessenmaximierung dem Kauf einer Staatsbürgerschaft zugrunde, muss wohl angenommen werden, dass der "Erwerber“ in Krisenzeiten im Zweifel eher auf seine Investition verzichten oder aber versuchen wird, diese so schnell wie möglich „rückabzuwickeln“, um seinen staatsbürgerlichen Pflichten nicht nachkommen zu müssen, seien sie beispielsweise fiskalischer oder militärischer Natur. ${ }^{74}$

Geht man davon aus, dass der Hauptzweck eines staatlichen Gemeinwesens das Wohl seiner Mitglieder ist, etwa durch Sicherung individueller Freiheiten, Aufrechterhaltung der öffentlichen Sicherheit, Ermöglichung gemeinsamer Teilhabe an gesellschaftlichen Entscheidungsprozessen etc., dann sind Staatsangehörige Teil eines komplexen politischen und sozialen wechselseitigen Beziehungsgeflechts, das die Bindung zwischen dem Individuum und dem Staat eindeutig von Produzent-Verbraucher- oder Angebot-Nachfrage-Beziehungen unterscheidet. ${ }^{75}$ Dieser Sichtweise widerspricht jedoch die Einbürgerungspraxis vieler Staaten, die Staatsangehörigkeit und damit die politische Zugehörigkeit zunehmend in einen handelbaren Vermögenswert der globalen Marktwirtschaft zu verwandeln. „Citizenship by Invest-

72 „Goldene Pass“- und „Goldene Visa“-Programme bestehen in derzeit knapp 100 Staaten, vgl. Ayelet Shachar, Beyond Open and Closed Borders: the Grand Transformation of Citizenship, Jurisprudence 11 (2020), 1-27 (22).

73 Insoweit scheint der bekannte Ausspruch des römischen Kaisers Vespasian (Regierungszeit 69 bis $79 \mathrm{n}$. Chr.) pecunia non olet, also dass „Geld nicht stinke“ auch, wenn es durch eine Urin-Steuer eingenommen werde, für so manchen Staat heute auch auf den Verkauf der Staatsangehörigkeit übertragbar. Zum Zitat, vgl. auch Georg Büchmann, Geflügelte Worte, Frankfurt a. M. / Hamburg: Fischer 1957, 211.

74 So auch, Ayelet Shachar, Citizenship for Sale?, in: Shachar/Bauböck/Bloemraad/Vink (Fn. 9), 789-816 (807).

75 Vgl. Shachar (Fn. 74), 808. 
ment"-Programme, die teilweise mit Hochglanz-Videos vermarktet werden, bieten u. a. die karibischen Inselstaaten Antigua und Barbuda, ${ }^{76}$ Dominica, ${ }^{77}$ Grenada, ${ }^{78}$ St. Kitts und Nevis, ${ }^{79}$ aber auch die Türkei ${ }^{80}$ und die Republik Moldau an. ${ }^{81}$ Letztere wirbt explizit damit, mit ihrer Staatsbürgerschaft visafreien Zugang zu 122 Zielen, darunter den Ländern des SchengenRaums, zu ermöglichen.

Häufig wird St. Kitts und Nevis, das in den 80er Jahren ein „Goldener Pass"-Programm auflegte, als Erfinder solch ökonomisch-motivierter Einbürgerungen genannt. ${ }^{82}$ Doch der im Nottebohm-Urteil des IGH beschriebene Sachverhalt deutet auf ähnliche Modelle bereits in den 1930er Jahren hin. Der 1881 geborene deutsche Staatsangehörige Friedrich Nottebohm, seit 1905 wohnhaft in Guatemala, beantragte - einen Monat nach dem deutschen Überfall auf Polen im Oktober 1939 - die liechtensteinische Staatsbürgerschaft. ${ }^{83}$ Bis auf einen Bruder, der im Fürstentum lebte und den er gelegentlich besuchte, hatte Nottebohm keinerlei Bezug zu diesem Land. Nach dem damals geltenden Einbürgerungsrecht Liechtensteins war Voraussetzung zur Einbürgerung u. a. die Zusage der Aufnahme in den Heimatverband einer liechtensteinischen Gemeinde sowie ein mindestens dreijähriger Aufenthalt im Gebiet des Fürstentums - wobei von diesem Erfordernis in besonders berücksichtigungswürdigen Fällen ausnahmsweise abgesehen werden konnte. Schließlich sah das Gesetz auch vor, dass der Antragsteller verschiedene - von der Fürstlichen Regierung festzusetzende - Einbürgerungsgebühren zu entrichten habe. ${ }^{84}$ Ohne (erkennbare) besondere Gründe wurde Nottebohm aber von der dreijährigen Aufenthaltspflicht im Vorfeld einer Einbürgerung befreit und gegen Zahlung von 25.000 Schweizer Franken an eine Liechtensteiner Gemeinde sowie von 12.500 Schweizer Franken

76 Offizielle Citizenship by Investment Website von Antigua und Barbuda, <https:// cip.gov.ag/> (12.10.2021).

77 Offizielle Citizenship by Investment Website von Dominica, <https://cbiu.gov.dm/> (12.10.2021).

78 Offizielle Citizenship by Investment Website von Grenada, <https://www.cbi.gov.gd/> (12.10.2021).

79 Offizielle Citizenship by Investment Website von St. Kitts und Nevis, <https:// www.ciu.gov.kn/> (12.10.2021).

80 Offizielle Information der türkischen Regierung, <https://www.invest.gov.tr/> (12.10. 2021).

81 Offizielle Citizenship by Investment Website der Republik Moldau, <http://cbi.gov.md/> (12.10.2021).

82 Siehe beispielsweise, European Parliamentary Research Service, Citizenship by Investment and Residency by Investment Schemes in the EU, PE 627.128 - October 2018, 11. Siehe auch, Shachar (Fn. 74), 794.

83 Ausführlich zum Sachverhalt, Makarov (Fn. 42), 407 ff.; Loewenfeld (Fn. 43), 387 ff.

84 IGH, Nottebohm (Fn. 10), 13 f. 
an das Fürstentum zuzüglich der Verfahrensgebühren und der Verpflichtung zur Zahlung einer jährlichen „Einbürgerungssteuer“ in Höhe von 1.000 Schweizer Franken im Fürstentum Liechtenstein eingebürgert. ${ }^{85}$ Ein Vorgang, der aus heutiger Sicht wie eine Blaupause für spätere „Goldene Pass“Programme wirkt.

Diese nach liechtensteinischem Recht wirksame Einbürgerung half Nottebohm jedoch nicht. 1940 kehrte er in das zu dem Zeitpunkt noch neutrale Guatemala zurück. Dort wurde er 1943 als „Angehöriger eines verfeindeten Staates" - Guatemala befand sich seit 1941 im Krieg mit dem Deutschen Reich - verhaftet, in die Vereinigten Staaten von Amerika (USA) überführt und sein Vermögen konfisziert. Nach seiner Freilassung im Jahre 1946 kehrte er nach Liechtenstein zurück, das u. a. wegen dieser Enteignung im Wege des diplomatischen Schutzes Entschädigungsansprüche gegenüber Guatemala geltend machte. Der IGH sprach Guatemala aber zu, aufgrund des Fehlens jeglicher Verbundenheit zu Liechtenstein, die Einbürgerung Nottebohms nicht anerkennen zu müssen und Liechtenstein zugleich dessen diplomatischen Schutz verwehren zu dürfen. ${ }^{86}$

Doch während die „Goldene Pass"-Vergabe - wie im Fall Nottebohm - in der Regel einem individuellen, strategischen Staatsangehörigkeitserwerb oder -wechsel dient, führen nicht alle „Citizenship by Investment“-Programme zu "Goldenen Pässen“. Vielmehr können sie potenziell auch dazu genutzt werden, sich unliebsamer Einwohner dadurch zu entledigen, dass man ihre Einbürgerung und Fragen des staatsbürgerlichen Status externalisiert und statt individueller Staatsangehörigkeits-Deals großangelegte Kaufprogramme für bestimmte Bevölkerungsteile initiiert, wie das Beispiel Kuwaits und der Vereinigten Arabischen Emirate (VAE) zeigt. Diese beiden arabischen Staaten verhandelten mit dem ostafrikanischen Inselstaat der Komoren den Verkauf der komorischen Staatsangehörigkeit für die in Kuwait und den VAE lebenden Staatenlosen, sog. „Bidoons“.87 Ziel war es dabei nicht, die „Bidoons“ auf die Komoren überzusiedeln, sondern ihren Status der Staatenlosigkeit zu

85 IGH, Nottebohm (Fn. 10), $15 \mathrm{f}$.

86 IGH, Nottebohm (Fn. 10), 26.

87 Vgl. Abu Sulaib, Stateless 'Bidoon' in Kuwait: a Crisis of Political Alienation, Middle E. Stud. 57 (2021), 134-150 (140); siehe auch Caia Vlieks/Ernst Hirsch Ballin/Maria José Recalde Vela, Solving Statelessness: Interpreting the Right to Nationality, NQHR 35 (2017), 158-175 (159f.). Siehe schließlich auch David Lewis/Ali Amir Abmed, Comoros Passport Scheme Was Unlawful, Abused by ,Mafia' Networks - Report, Reuters, 23.3.2018. Dieser Fall unterscheidet sich daher von der sog. russischen "Passportisierung“ in der Ost-Ukraine, der massenhaften Verleihung der Staatsangehörigkeit an Personen mit einer anderen Staatsangehörigkeit und Wohnsitz im Ausland. Denn eine solche berührt unmittelbar die Souveränität des Staates der früheren Staatsangehörigkeit, indem diesem u. a. die Möglichkeit diplomatischen Schutzes der (früheren) Staatsbürger genommen wird, ausführlich Peters (Fn. 29). 
überwinden, ohne ihnen (sofort) die eigene also kuwaitische oder VAEStaatsbürgerschaft zu verleihen. ${ }^{88}$

Wenn die Komoren einer großen Gruppe von Menschen, die keinerlei Verbindung zu diesem Staat haben, die Staatsangehörigkeit gegen Zahlungen eines anderen Staates übertragen, ist das zunächst ein rein innerstaatlich relevanter Sachverhalt, zumindest in dem Maße, wie die Komoren nicht versuchen, die nominelle Staatsangehörigkeit gegenüber anderen Staaten, etwa im Wege diplomatischen Schutzes, durchzusetzen. Denn dann würde wieder die „Nottebohm“-Schwelle des IGH greifen, die eine „echte“ Verbindung erfordert. Die Komoren dürften aber kein nennenswertes Interesse daran haben, diplomatische Ressourcen für Bürger aufzuwenden, zu denen sie - bis auf das in diesem Fall rein formale Band der Staatsangehörigkeit - keinerlei Verbindung haben. ${ }^{89}$

\section{Goldene Pässe in der Europäischen Union}

Das Phänomen von „Goldenen Pass“-Programmen hat vor den Toren der EU nicht Halt gemacht. Laut einer Studie im Auftrag des Europäischen Parlaments aus dem Jahr 2018 bietet die große Mehrheit der EU-Mitgliedstaaten Nicht-EU-Bürgern die Möglichkeit, sich gegen eine finanzielle Investition einbürgern zu lassen. ${ }^{90}$ Die Voraussetzungen variieren jedoch stark von Mitgliedstaat zu Mitgliedstaat. Reine „Goldene Pass“-Programme, bei denen kein (längerer) Aufenthalt im Staatsgebiet oder die Begründung eines Wohnsitzes erforderlich waren, mithin eine Verbundenheit nahezu ausschließlich durch die finanzielle Zuwendung begründet werden kann, bestanden zuletzt allein in den drei Mitgliedstaaten Bulgarien, Malta und Zypern, die diese Programme mit sehr unterschiedlichen Voraussetzungen zwischen 2005 und 2013 einführten. ${ }^{91}$

So war in Bulgarien eine Investition in Höhe von 1 Mio. EUR erforderlich. In Malta galt als Voraussetzung die Einzahlung von 650.000 EUR in einen nationalen Investmentfond, eine Investition auf der Insel in Höhe von mindestens 150.000 EUR sowie der Nachweis über das Eigentum an

88 Atossa Araxia Abrabamian, The Bizarre Scheme to Transform a Remote Island Into the New Dubai, The Guardian, 11.11.2015. Voraussetzung für die völkerrechtliche Wirksamkeit solcher Einbürgerungen ist aber zumindest auch die Zustimmung der betroffenen Individuen, vgl. Peters (Fn. 29).

89 Vgl. auch Peter J. Spiro, Kuwait Bulk-Orders Comoros Citizenship for Stateless Bidoon, Opinio Juris, 13.11.2014.

90 European Parliamentary Research Service (Fn. 82), $12 \mathrm{ff.}$

91 EU-Kommission (Fn. 3), 3 ff. 
einer Immobilie auf Malta im Wert von mindestens 350.000 EUR bzw. einer Mietimmobilie mit einer Jahresmiete von mindestens 16.000 EUR. ${ }^{92}$ Zypern verlangte Investitionen von mind. 2,5 Mio. EUR, was laut Zyperns Präsident Anastasiades dem - von der internationalen Finanzkrise gebeutelten - Inselstaat Investitionen im Wert von 9,7 Mrd. EUR bescherte, ${ }^{93}$ im Gegenzug zu etwa 3500, vornehmlich an Russen und Chinesen, vergebenen "Goldenen Pässen“. ${ }^{94}$ An diese kommerzialisierte Schnell-Einbürgerung war in keinem der drei Staaten die Voraussetzungen eines tatsächlichen Wohnsitzes geknüpft. Im Zweifel reichte gar eine einmalige Stippvisite im jeweiligen Land, um die Staatsbürgerschaft dauerhaft zu erwerben. ${ }^{95}$

Nachdem Malta 2013 sein „Citizenship by Investment“-Programm vorgelegt hatte, forderte das Europäische Parlament die EU-Kommission auf, tätig zu werden. ${ }^{96}$ Auf Druck der EU-Kommission führte Malta dann die Voraussetzung eines Wohnsitznachweises von mindestens zwölf Monaten vor der Einbürgerung ein, ohne dass jedoch ein entsprechender Aufenthalt in Malta vonnöten war. ${ }^{97}$ Vielmehr genügte in der Praxis eine Aufenthaltsgenehmigung, für die kein Wohnsitz erforderlich ist, oder das Vorlegen von Bordpässen, die Zahlung von Einkommenssteuer auf Malta, Nachweise über gemeinnützige Spenden oder etwa Mitgliedschaften in örtlichen Sportvereinen. ${ }^{98}$ Ähnliche Voraussetzungen für eine Einbürgerung führte auch $\mathrm{Zy}$ pern im Jahr 2016 ein. ${ }^{99}$ Kenntnisse der Landessprache oder eine anderweitige Verbundenheit waren ebenfalls nicht erforderlich. Laut EU-Kommission hielten die zypriotischen Behörden vielmehr "die in Zypern getätigte Investition als solche bereits für eine ausreichende Verbundenheit des Antragstellers zu Zypern"“. ${ }^{100}$ Nach erheblicher Kritik hat Zypern die Vergabepraxis nun im November 2020 vorerst gestoppt. ${ }^{101}$

92 Ausführlich hierzu, EU-Kommission (Fn. 3), 4.

93 Die Informationslage bzgl. vergebener „Goldener Pässe“ bleibt insgesamt sehr dünn und die EU-Mitgliedstaaten ziemlich intransparent, was diese konkreten Vergabezahlen angeht, vgl. European Parliamentary Research Service (Fn. 82), $17 \mathrm{ff}$.

94 Zyperns Präsident rechtfertigt Vergabe „Goldener Pässe” (Fn. 2).

95 Ausführlich hierzu, EU-Kommission (Fn. 3), 4.

96 Europäisches Parlament, Entschließung zum Verkauf der Unionsbürgerschaft (2013/ 2995(RSP)) v. 14.1.2014.

97 European Parliamentary Research Service (Fn. 82), 20.

98 EU-Kommission (Fn. 3), $7 \mathrm{f}$.

99 EU-Kommission (Fn. 3), $7 \mathrm{f}$.

100 EU-Kommission (Fn. 3), 5.

101 Zyperns Präsident rechtfertigt Vergabe „Goldener Pässe” (Fn. 2). 


\section{Vereinbarkeit mit dem Europarecht?}

"Goldene Pässe“ sind schon für sich genommen ein interessantes Phänomen, das grundlegende Fragen der Staatlichkeit aufwirft. Innerhalb der EU ergibt sich aber eine zusätzliche Besonderheit aufgrund der engen Verschränkung der Mitgliedstaaten. Da zu der bulgarischen, maltesischen und zypriotischen Staatsangehörigkeit gem. Art. 9 S. 2 und 3 EUV und Art. 20 AEUV die Unionsbürgerschaft hinzutritt, und neu-Eingebürgerte somit automatisch in den Genuss aller Rechte der Unionsbürger gelangen, hat die zunächst rein innerstaatliche Vergabe "Goldener Pässe“ an Drittstaatsangehörige potenziell Auswirkungen in der gesamten EU. Der inhärente ideelle Wert der Unionsbürgerschaft, der nicht nur aus den wirtschaftlichen sondern auch aus den aus ihr erwachsenen politischen Statusrechten herrührt, könnte durch diese Ökonomisierung in seiner Substanz gefährdet werden. ${ }^{102}$ Dabei handelt es sich jedoch in erster Linie um theoretische Überlegungen, denn die tatsächlichen Auswirkungen „Goldener Pässe“ auf die knapp 450 Mio. Einwohner der EU sind bislang (noch) marginal.

Das Europäische Parlament hat dennoch bereits im Jahr 2014 seine Besorgnis darüber zum Ausdruck gebracht, dass nationale Programme, die den "direkten oder unverhohlenen Verkauf“ der Unionsbürgerschaft mit sich bringen, die Idee der Unionsbürgerschaft an sich untergraben könnten und zugleich die EU-Kommission aufgefordert, die verschiedenen Staatsbürgerschaftsregelungen im Lichte der europäischen Werte, des Geistes und des Wortlauts des EU-Rechts zu bewerten. ${ }^{103}$ Denn der Verkauf der Unionsbürgerschaft könnte das gegenseitige Vertrauen erschüttern, auf dem die Union gegründet ist. ${ }^{104}$ Am 20. Oktober 2020 hat die EUKommission nun Vertragsverletzungsverfahren gegen Zypern und Malta wegen ihrer "Goldenen Pass“-Programme eingeleitet. ${ }^{105}$ Aus Sicht der EU-Kommission ist die „Gewährung der Staatsangehörigkeit - und damit der Unionsbürgerschaft - durch diese Mitgliedstaaten gegen eine im Voraus festgelegte Zahlung oder Investition und ohne eine echte Verbindung zu den betreffenden Mitgliedstaaten nicht mit dem in Art. 4 Abs. 3 EUV verankerten Grundsatz der loyalen Zusammenarbeit vereinbar", sondern untergräbt im Gegenteil die Integrität des Status der Unionsbürgerschaft. ${ }^{106}$

\footnotetext{
102 So auch, Hilpold (Fn. 37), 1072.

103 Europäisches Parlament (Fn. 96).

104 Europäisches Parlament (Fn. 96).

105 EU-Kommission (Fn. 2).

106 EU-Kommission (Fn. 2).
} 
Die Position der EU-Kommission lässt sich auch anhand der ökonomischen „theory of clubs" 107 und damit außerhalb des europarechtlichen Rahmens verdeutlichen. Die „theory of clubs“ versteht Staaten als „Vereine“, deren „Mitglieder“ die Staatsbürger sind, die wiederum „Mitgliedsbeiträge“ (im Sinne staatsbürgerlicher Pflichten, insbesondere Steuern) zahlen und im Gegenzug von den Vereinsvorteilen (staatsbürgerliche Rechte, Raum der Sicherheit, der Freiheit und des Rechts) profitieren, die ihnen als Mitglieder zur Verfügung gestellt werden. Der Verein bestimmt die Bedingungen zur Aufnahme neuer Mitglieder und übt dieses Recht im Interesse der bisherigen Vereinsmitglieder aus. Bleibt man in diesem Bild, stellt die EU einen „Meta-Verein“ oder „Dachverband“ dar, in dem sich verschiedene Vereine zusammengeschlossen haben. Der „Dachverband“ EU ist Anbieter von Vereinsleistungen, die von seinen Mitgliedern - den EU-Mitgliedstaaten finanziert und diesen zur Verfügung gestellt werden. Wie andere Vereine auch, hat der „Dachverband“ eine Governance-Struktur, die auch die Regeln und Verfahren für Aufnahme und Austritt von Vereinsmitgliedern festlegt, kodifiziert etwa in Art. 49 und Art. 50 EUV. Letztlich sind Geldgeber und Nutznießer des „Dachverbands“ aber die Mitglieder der einzelnen Vereine, also die Staatsbürger der Mitgliedstaaten. Behalten sich die Vereine vor, den Zutritt neuer Mitglieder selbst zu bestimmen und profitieren zugleich alle Vereinsmitglieder automatisch von den Vorzügen des „Dachverbands“, entstehen dadurch Externalitäten: Während die „Beitrittsgebühren " für neue Vereinsmitglieder nur an den Verein gehen, profitieren die neuen Vereinsmitglieder auch von den Leistungen des „Dachverbands“, der nicht nur von dem Verein, dem sie beigetreten sind, finanziert wird, sondern auch von den Mitgliedern der übrigen im „Dachverband“ organisierten Vereine. Der wissenschaftliche Dienst des Europaparlaments bezeichnet die Mitgliedstaaten, die ihre Staatsbürgerschaft vermarkten, daher als „Trittbrettfahrer“, 108 die von der Attraktivität der EU und anderer Mitgliedstaaten (mit)profitieren, ohne die „Vorteile“ ihrer „Goldenen Pass“Programme zu teilen.

Dies wirft die grundlegende Frage auf, inwieweit der „Dachverband“ EU seinen Teilvereinen Regeln zum Vereinsbeitritt vorschreiben können sollte. Denn im „Dachverband“ EU kollidieren zwei grundlegende Rechte: Zum einen das Recht jedes Vereinsmitglieds sich im Sinne der Grundfreiheiten und des Freizügigkeitsrechts zwischen den verschiedenen Vereinen frei zu bewegen, zum anderen das Recht jedes einzelnen Vereins, die eigenen Vereinsmitgliedschafts-Voraussetzungen, also die Voraussetzungen an die Staats-

$107 \mathrm{Konrad} /$ Rees (Fn. 4), 2.

108 European Parliamentary Research Service (Fn. 82), 23. 
bürgerschaft, frei zu bestimmen. ${ }^{109}$ Föderale Staaten wie die USA oder die Bundesrepublik Deutschland können auch als „Dachverband“ verstanden werden. ${ }^{110}$ In diesen wird das Staatsbürgerschaftsrecht aber ausschließlich auf der Meta-Ebene bestimmt. Anders als diese ist die EU jedoch gerade kein Staat, sondern hat einen sui generis Charakter, vom Bundesverfassungsgericht treffend als „Staatenverbund" ${ }^{111}$ bezeichnet. ${ }^{112}$

Die Notwendigkeit, das Recht der Staatsbürgerschaft europarechtlich zu beschränken bzw. zu harmonisieren, könnte sich also aus der Kollision der Einbürgerungs-Praxis mit dem Europarecht ergeben. Ins Feld geführt werden diesbezüglich häufig das inhärente Potenzial „Goldener Pass“-Programme für Korruption, Geldwäsche und Steuerhinterziehung. ${ }^{113}$ In diesem Bereich sind bereits sekundärrechtliche Regelungen wie die GeldwäscheRichtlinie $^{114}$ und die Richtlinie zur Verwaltungszusammenarbeit in Steuerfragen ${ }^{115}$ in Kraft. Der wissenschaftliche Dienst des Europäischen Parlaments hat auch vorgeschlagen, dass die EU-Kommission die mitgliedstaatlichen Hintergrund-Überprüfungen potenzieller Antragsteller „Goldener Pässe" kontrollieren sollte, und hat zudem eine stärkere Transparenz der Mitgliedstaaten mit Blick auf „Goldene Pass“-Programme angemahnt. ${ }^{116}$ Diese Ansätze berühren jedoch nicht den Kern des Problems „Goldener Pässe“, sondern können allenfalls negative Nebeneffekte solcher Programme beschränken.

\section{Grundsatz der loyalen Zusammenarbeit}

Die EU-Kommission hat sich bei der Einleitung der Vertragsverletzungsverfahren gegen Malta und Zypern daher auf einen Verstoß gegen den Grundsatz der loyalen Zusammenarbeit aus Art. 4 Abs. 3 EUV i.V.m. der Unionsbürgerschaft gem. Art. 20 AEUV berufen. Aus Sicht

109 Vgl. Konrad/Rees (Fn. 4), 3.

110 Vgl. Konrad/Rees (Fn. 4), 2.

111 Vgl. BVerfGE, Lissabon (Fn. 50), (Rn. 229).

112 Zum sui generis Charakter der Europäischen Union, siehe auch überblicksartig Julien Berger, International Investment Protection Within Europe, Oxford: Routledge 2021, $88 \mathrm{ff}$.

113 Ausführlich: European Parliamentary Research Service (Fn. 82), $28 \mathrm{f}$.

114 Richtlinie (EU) 2015/849 des Europäischen Parlaments und des Rates v. 20.5.2015 zur Verhinderung der Nutzung des Finanzsystems zum Zwecke der Geldwäsche und der Terrorismusfinanzierung.

115 Richtlinie (EU) 2011/16 des Rates v. 15.2.2011 über die Zusammenarbeit der Verwaltungsbehörden im Bereich der Besteuerung.

116 European Parliamentary Research Service (Fn. 82), 49 ff. 
der EU-Kommission untergräbt die „Gewährung der Unionsbürgerschaft für im Voraus festgelegte Zahlungen oder Investitionen ohne echte Verbindung zu den betreffenden Mitgliedstaaten den Wesensgehalt der Unionsbürgerschaft". ${ }^{117}$ Denn erfolgt eine Einbürgerung in einem EU-Mitgliedstaat allein auf Grundlage einer Geld-Zahlung, ohne dass eine weitergehende echte Bindung besteht, stellt dies aus Sicht der EU-Kommission eine nicht hinnehmbare Abweichung von der herkömmlichen Einbürgerung dar. Da EU-Mitgliedstaaten jedoch nicht die Rechte eines Unionsbürgers, der in einem anderen Mitgliedstaat auch ohne Bindung zu diesem eingebürgert worden ist, beschränken dürfen, muss aus Sicht der EU-Kommission nach dem Grundsatz der loyalen Zusammenarbeit aus Art. 4 III EUV „jeder Mitgliedstaat dafür sorgen, dass die Staatsbürgerschaft nicht ohne echte Bindung zum Land oder dessen Bürgern verliehen wird". ${ }^{118}$

In ähnlicher Weise hatte bereits Generalanwalt Maduro im Fall Rottmann argumentiert. Dieses Verfahren betraf die Frage, ob die deutsche Einbürgerung von Herrn Rottmann, einem gebürtigen Österreicher, der sich die deutsche Staatsangehörigkeit - durch das Verschweigen von gegen ihn anhängigen Strafverfahren - erschlichen hatte, wieder zurückgenommen werden konnte, selbst wenn dies seine Staatenlosigkeit zur Folge hätte. In seinen Schlussanträgen stellte Generalanwalt Maduro fest, dass der Grundsatz der loyalen Zusammenarbeit bzw. die Unionstreue ${ }^{119}$

„dann berührt sein könnte, wenn ein Mitgliedstaat unter Verstoß gegen geltendes Recht auf massive Weise die Einbürgerung von Drittstaatsangehörigen betriebe, ohne zuvor die Kommission oder seine Partner zu konsultieren. “120

Der Grundsatz der loyalen Zusammenarbeit ist ein „fundamentales Verfassungsstrukturprinzip der EU ${ }^{121}$ bzw. eine wesentliche Grundlage der Unionsrechtsordnung. ${ }^{122}$ Ein Verstoß gegen diesen Grundsatz beeinträchtigt aus Sicht des EuGH die Union „bis in ihre Grundfesten“.'23 Hintergrund ist, dass die EU als Rechtsunion, die anders als ein Staat nicht über ein Gewaltmonopol verfügt, „im Interesse der Einheit und praktischen Wirksamkeit“ ihres Rechts „auf dessen unionsrechtskonforme normative, administrative

117 EU-Kommission (Fn. 2). Vgl. auch mit ähnlicher Position, Hilpold (Fn. 37), 1074.

118 EU-Kommission (Fn. 3), 7.

119 Die Begrifflichkeiten werden synonym verwendet, vgl. Christian Calliess/Wolfgang Kabl/Adelheid Puttler, in: Christian Calliess/Matthias Ruffert, EUV/AEUV, 5. Aufl., München: C. H. Beck 2016, Art. 4 EUV, Rn. 29.

120 EuGH, Schlussanträge des Generalanwalts Maduro - Rottmann (Fn. 48), Rn. 30.

121 M. w. N. Calliess/Kabl/Puttler (Fn. 119), Rn. 34.

122 EuGH, Rs. C-101/91, Rn. 23.

123 EuGH, Rs. 39/72, ECLI:EU:C:1973:13, Rn. 25. 
und gerichtliche Durchführung" durch die Mitgliedstaaten existenziell angewiesen ist. ${ }^{124}$ Aufgrund seiner begrifflichen Weite bedarf der Grundsatz der loyalen Zusammenarbeit stets einer Konkretisierung durch andere Normen wie etwa vorliegend Art. 20 AEUV, um pflichtenerzeugend zu wirken und justiziabel zu sein. ${ }^{25}$ Auf Art. 20 AEUV hat der EuGH bereits sein Rottmann-Urteil gestützt, in welchem er feststellte, dass „angesichts der Bedeutung, die das Primärrecht dem Unionsbürgerstatus beimisst“, bei der Überprüfung einer staatlichen Maßnahme wie dem Entzug der Staatsangehörigkeit die möglichen Folgen zu berücksichtigen seien, „die diese Entscheidung für den Betroffenen und gegebenenfalls für seine Familienangehörigen in Bezug auf den Verlust der Rechte, die jeder Unionsbürger genießt, mit sich bringt“. ${ }^{126}$ Auf den Fall einer Einbürgerung aufgrund eines „Goldener Pass“Programms scheint dieser Rechtsgedanke jedoch nicht übertragbar. Denn mit dieser geht ja gerade kein Verlust von unionsbürgerlichen Rechten, sondern vielmehr ein Zugewinn an solchen einher. Mit Blick auf die Einbürgerung sind dem Unionsrecht daher auch grundsätzlich keine Vorgaben zu entnehmen. ${ }^{127}$

Der EuGH hat sich jedoch - ebenfalls in Rottmann - explizit eine weitergehende Prüfung auch bei der Einbürgerung vorbehalten:

„Der Vorbehalt, dass das Unionsrecht zu beachten ist, berührt nicht den vom Gerichtshof [...] anerkannten [...] Grundsatz des Völkerrechts, wonach die Mitgliedstaaten für die Festlegung der Voraussetzungen für den Erwerb und den Verlust der Staatsangehörigkeit zuständig sind, sondern stellt den Grundsatz auf, dass im Fall von Unionsbürgern die Ausübung dieser Zuständigkeit, soweit sie die von der Rechtsordnung der Union verliehenen und geschützten Rechte berührt [...], der gerichtlichen Kontrolle im Hinblick auf das Unionsrecht unterliegt. "

Eine gerichtliche Kontrolle auch von Erwerbsvorgängen scheint daher möglich zu sein. Dabei muss der EuGH jedoch die Grenzen der europäischen Integration im Blick behalten.

\section{Grenzen Europäischer Integration}

Mangels Kompetenzzuweisung an die EU in den europäischen Verträgen, verbleibt das Staatsangehörigkeitsrecht Sache der Mitgliedstaaten. Sollte sich

124 M. w. N. Calliess/Kabl/Puttler (Fn. 119), Rn. 35.

125 Vgl. m. w. N. Calliess/Kabl/Puttler (Fn. 119), Rn. 44.

126 EuGH, Rottmann (Fn. 11), Rn. 56. Siehe auch Schönberger (Fn. 53), Rn. 45.

127 Schönberger (Fn. 53), Rn. 46.

128 EuGH, Rottmann (Fn. 11), Rn. 48. 
der EuGH - etwa im Rahmen der Ende 2020 initiierten Vertragsverletzungsverfahren gegen Malta und Zypern - mit „Goldenen Pässen“ beschäftigen, wird er im Fahrwasser nationalen Staatsangehörigkeitsrechts äußerst vorsichtig navigieren müssen. Der Gerichtshof steht gewissermaßen vor der Quadratur des Kreises: Der mitgliedstaatlichen Praxis der Goldenen Pässe einen Riegel vorzuschieben, ohne in unzulässiger Weise in das nationale Staatsangehörigkeitsrecht einzugreifen. Deutliche Beschränkungen der mitgliedstaatlichen Souveränität - insbesondere mit Blick auf die Frage, wer unter welchen Voraussetzungen eingebürgert werden darf - könnten schnell als Kompetenzüberschreitung des EuGH verstanden werden und sogar die nationalen Verfassungsidentitäten berühren. Denn hierdurch griffe dieser eindeutig in die Bestimmungen über das Staatsvolk ein und könnte - quasi durch die Hintertür - einen weiteren Integrationsschritt zu einem europäischen Bundesstaat gehen, indem er die Unionsbürgerschaft als die nationale Staatsbürgerschaft überlagernd betrachten würde. Außerhalb formeller Vertragsänderungen dürfte eine solche Interpretation ein rechtspolitisch gefährliches Unterfangen darstellen. Das Bundesverfassungsgericht (BVerfG) beispielsweise hat sich für derartige Fälle die „ultra-vires-“ und „Identitätskontrolle“ vorbehalten. ${ }^{129}$ Die von den Karlsruher Richterinnen und Richtern in ihrem Lissabon-Urteil aufgestellten Grenzen würden jedenfalls überschritten, was das BVerfG zum Handeln zwingen dürfte:

„Auch angesichts des Ausbaus der Rechte der Unionsbürger bewahrt das deutsche Staatsvolk solange seine Existenz, wie die Unionsbürgerschaft die Staatsangehörigkeit der Mitgliedstaaten nicht ersetzt oder überlagert. Der abgeleitete Status der Unionsbürgerschaft und die Wahrung der mitgliedstaatlichen Staatsangehörigkeit bilden die Grenze für die in Art. 25 Abs. 2 AEUV angelegte Entwicklung der Unionsbürgerrechte und für die Rechtsprechung des Gerichtshofs der Europäischen Union. So bestehen mitgliedstaatliche Möglichkeiten der Differenzierung aufgrund der Staatsangehörigkeit fort. [...]“130

Ähnlich dürften auch die Reaktionen in anderen Mitgliedstaaten ausfallen. Eine neue, diesmal europarechtlich geprägte, Nottebohm 2.0-Entscheidung des EuGH, ist auch nur schwer vorstellbar. Denn die Entflechtung von Unionsbürgerschaft und mitgliedstaatlicher Staatsangehörigkeit ist in den Europäischen Verträgen gerade nicht vorgesehen und würde zudem zu einem zwei-Klassen-System der Unionsbürgerschaft und großer Rechtsunsicherheit im innereuropäischen Verkehr führen. Wenn aber eine gerichtliche Einschränkung von „Goldenen Pass“-Programmen aufgrund der mitgliedstaatli-

129 Zur ultra-vires und Identitätskontrolle, vgl. auch Ulrich Haltern, Ultra-vires-Kontrolle im Dienst europäischer Demokratie, NVwZ 39 (2020), 817-823.

130 Vgl. BVerfGE, Lissabon (Fn. 50), (Rn. 350). 
chen Souveränität in Staatsangehörigkeitsfragen sowie der Verknüpfung von Unionsbürgerschaft und mitgliedstaatlicher Staatsangehörigkeit nicht möglich ist, bliebe nur der Weg über eine Vertragsänderung gem. Art. 48 EUV. Eine (teilweise) Kompetenzzuweisung an die EU zur Harmonisierung des Staatsbürgerschaftsrechts würde einen weiteren (großen) Schritt hin zu einem europäischen Bundesstaat darstellen und erscheint daher - zum jetzigen Zeitpunkt - politisch nicht durchsetzbar.

Auch die Nottebohm-Rechtsprechung des IGH ist auf den EU-Kontext nicht übertragbar. Der IGH hat - wie oben gezeigt - den Begriff der „besonderen Verbundenheit“ geprägt, um aufzuzeigen, dass zwar ein Staat frei über die Vergabe der Staatsangehörigkeit verfügen kann, wenn er diese gegenüber anderen Staaten (insbesondere im Rahmen diplomatischen Schutzes) geltend macht, jedoch eine solche Verbundenheit bestehen muss, da andernfalls der andere Staat die Anerkennung der Staatsbürgerschaft bzw. des diplomatischen Schutzes verweigern kann. EU-Mitgliedstaaten sind jedoch von Unionsrechts wegen verpflichtet, Staatsbürger anderer EU-Mitgliedstaaten, also Unionsbürger, nicht zu diskriminieren, und müssen daher die Staatsbürgerschaft anderer Mitgliedstaaten anerkennen. ${ }^{131}$ Eine nachträgliche Kontrolle der Einbürgerungsvoraussetzungen anderer EU-Mitgliedstaaten durch nationale Gerichte ist also ausgeschlossen, sodass es schon nicht zu einer Überprüfung der Verbundenheit kommen kann.

Mithin bleibt statt einer juristischen nur die politische Lösung, also der einfache politische Druck der übrigen Mitgliedstaaten, der Erosion der Unionsbürgerschaft durch strengere Einbürgerungsregeln entgegenzutreten.

\section{Ausblick}

Der Verkauf der traditionell unverkäuflichen Staatsbürgerschaft kann gesellschaftliche Bindungen und die Auffassung davon, was es bedeutet, Teil einer politischen Gemeinschaft zu sein, untergraben. Entsprechende Programme stehen auch im Widerspruch zu den grundlegenden Werten der EU, die in Art. 2 EUV explizit aufgezählt werden. Denn während die Tendenz innerhalb der EU eher in Richtung strengerer Einwanderungsregeln geht, ${ }^{132}$ schaffen „Goldene Pass“-Programme privilegierte Wege zur Erlangung der Unionsbürgerschaft. Flüchtende zahlen teilweise Unsummen an Schlepper und nehmen Lebensgefahren auf sich, um nach Europa zu gelangen, stets mit dem Risiko, an der europäischen Außengrenze zurückgewiesen zu werden.

131 Vgl. auch Schönberger (Fn. 53), Rn. 40.

132 Vgl. European Parliamentary Research Service (Fn. 82), 5. 
Potenzielle Asylbewerber werden gezwungen, in die EU einzureisen, um einen Asylantrag zu stellen, Drittstaatsangehörigen, die seit Jahren in EUStaaten leben und inzwischen enge Verbundenheit zu diesen aufgebaut haben, wird teilweise die Einbürgerung verwehrt, während gleichzeitig auf Aufenthaltsvoraussetzungen zur Einbürgerung im Rahmen von „Citizenship by Investment"-Programmen verzichtet wird. Diejenigen, die vor Gewalt und Verfolgung im Ausland fliehen, haben oft keine andere Möglichkeit, als ihr Leben zu riskieren und den gefährlichen Weg in potenziell sichere Staaten auf sich zu nehmen, um Asyl oder ein Bleiberecht zu beantragen. Zugleich genießen andere, die es sich „leisten“ können, die Voraussetzungen „Goldener Pass"-Programme zu erfüllen, das Privileg schneller und oft unkomplizierter Einbürgerung, ohne Asylgründe vorweisen zu müssen oder sich zuvor im Zielland aufgehalten zu haben. ${ }^{133}$ "Goldene Pass"-Programme führen mithin zu einer weiteren Zementierung und Verschärfung bestehender - durch die Geburt determinierter und an die Staatsangehörigkeit anknüpfender globaler Ungleichheiten.

Entsprechende Programme europäischer Staaten sind jedoch, wie oben gezeigt, ohne Änderung der Europäischen Verträge rechtlich nur schwer zu beanstanden, auch wenn sie direkte Auswirkungen auf die Unionsbürgerschaft haben. Politischer Druck anderer Mitgliedstaaten erscheint insofern das einzig erfolgsversprechende Mittel.

\section{Summary: Citizenship as a Commodity - of Golden Passports and the European Union}

"Golden passport" schemes are increasingly gaining popularity around the world. Meanwhile, this trend has also reached the European Union. It now threatens to lead to a partial commercialisation of both national citizenship and the European citizenship. This contribution examines the evolution of national citizenship law through "golden passports" and addresses the question of the compatibility of such programs with the law of the European Union. It thereby reveals the difficulty of reconciling the sovereignty of member states in matters of nationality with the principle of sincere cooperation in the EU.

133 Vgl. auch Shachar (Fn. 72), 22. 